www.nature.com/clinicalpractice/endmet

previously. The four new loci identified in the present study contain a zinc-transporter gene that is expressed exclusively in pancreatic $\beta$ cells and involved in the final stage of insulin synthesis, and two genes that have a possible role in the development of the pancreas.

The authors conclude that genome-wide analyses can reveal previously unknown details of the pathogenesis of complex diseases, such as type 2 diabetes, and might contribute to new therapeutic approaches.

Original article Sladek R et al. (2007) A genome-wide association study identifies novel risk loci for type 2 diabetes. Nature 445: 881-885

\section{New algorithm to predict cardiovascular risk in women}

Algorithms to predict cardiovascular risk in women have remained largely unchanged for the past few decades, despite improved understanding of atherothrombosis. Ridker and colleagues, therefore, developed and validated a cardiovascular risk algorithm for women, which is based on a combination of traditional and new risk factors (alternative lipid measures, inflammatory biomarkers and markers of glycemic control).

The authors assessed 24,558 participants (aged $\geq 45$ years) without cancer or cardiovascular disease for 35 risk factors. Patients were randomly allocated to a derivation cohort $(n=16,400)$ whose data were used to devise the new risk algorithm, or to a validation cohort $(n=8,158)$ in which the new algorithm was used to predict patients' cardiovascular risk. These predictions were compared with those of currently used algorithms, and with incident cardiovascular events (myocardial infarction, stroke, coronary revascularization, or death) during follow-up (median 10.2 years).

The best-fitting (devised from nine risk factors) and simplified clinical (devised from a different group of eight risk factors) models accurately reclassified $40-50 \%$ of women traditionally considered to be at intermediate risk into higherrisk or lower-risk categories. The increased accuracy of cardiovascular risk prediction should allow lifestyle counseling and statin therapy to be targeted to those patients most likely to benefit from it, which will improve their cost:benefit ratio and minimize the incidence of treatment-related adverse effects.
The authors suggest that use of their simplified clinical model could have an immediate, positive effect on cardiovascular prevention strategies.

Original article Ridker PM et al. (2007) Development and validation of improved algorithms for the assessment of global cardiovascular risk in women: the Reynolds Risk Score. JAMA 297: 611-619

\section{Garlic and garlic supplements do not reduce moderate hypercholesterolemia}

Although the cholesterol-lowering effects of garlic and garlic supplements have been extensively advertised, their efficacy has not yet been proven in clinical trials. Gardner and colleagues conducted a randomized, placebo-controlled trial to evaluate the effects of raw garlic and two commercial garlic supplements in adults with moderate hypercholesterolemia.

Participants ( $n=192$, age $30-65$ years) with fasting plasma LDL-cholesterol levels of $3.36-4.91 \mathrm{mmol} / \mathrm{l}$ were randomly assigned to consume raw garlic, a powdered-garlic supplement (Garlicin ${ }^{\circledR}$, Nature's Way Products Inc., Springville, UT), an aged, garlic-extract supplement $\left(K_{\text {Kyolic }}{ }^{\circledR}\right.$, Wakunaga of America, Madero Mission Viejo, CA), or placebo 6 days a week, for 6 months. All garlic doses corresponded to about one clove per day. Levels of fasting plasma lipids, including LDL cholesterol, HDL cholesterol and triglycerides, were measured every month. Chemical content and stability of garlic components in the formulations were analyzed throughout the study.

By the end of the trial (completed by 169 participants), no clinically relevant changes were observed in fasting plasma lipid concentrations in any of the four study groups, and there were no significant differences between the groups. The authors noted that the major garlic components were at typical levels for each product and were stable throughout the trial, so their inefficacy was not attributable to poor product quality. The authors conclude that reasonable doses of garlic or garlic supplements cannot be recommended as cholesterol-lowering agents to adults with moderate hypercholesterolemia.

Original article Gardner CD et al. (2007) Effect of raw garlic vs commercial garlic supplements on plasma lipid concentrations in adults with moderate hypercholesterolemia: a randomized clinical trial. Arch Intern Med 167:346-353 